\title{
Retinal Spectral Image Analysis Methods Using Spectral Reflectance Pattern Recognition
}

\author{
G.M. Atiqur Rahaman ${ }^{1}$, Jussi Parkkinen ${ }^{2}$, Markku Hauta-Kasari ${ }^{3}$, \\ and Ole Norberg ${ }^{1}$ \\ ${ }^{1}$ DPC, Department of Applied Science and Design, Mid Sweden University, Sweden \\ ${ }^{2}$ School of Engineering, Monash University Sunway Campus, Malaysia \\ ${ }^{3}$ SIB Labs, School of Computing, University of Eastern Finland, Finland \\ atiqur.rahaman@miun.se, jussi@eng.monash.edu.my, \\ Markku.Hauta-Kasari@uef.fi, ole.norberg@miun.se
}

\begin{abstract}
Conventional 3-channel color images have limited information and quality dependency on parametric conditions. Hence, spectral imaging and reproduction is desired in many color applications to record and reproduce the reflectance of objects. Likewise RGB images lack sufficient information to successfully analyze diabetic retinopathy. In this case, spectral imaging may be the alternative solution. In this article, we propose a new supervised technique to detect and classify the abnormal lesions in retinal spectral reflectance images affected by diabetes. The technique employs both stochastic and deterministic spectral similarity measures to match the desired reflectance pattern. At first, it classifies a pixel as normal or abnormal depending on the probabilistic behavior of training spectra. The final decision is made evaluating the geometric similarity. We assessed several multispectral object detection methods developed for other applications. They could not proof to be the solution. The results were interpreted using receiver operating characteristics (ROC) curves analysis.
\end{abstract}

Keywords: Spectral reflectance image, Diabetic retinopathy, Spectral information divergence, ROC curves, Object detection, Objects classification.

\section{$1 \quad$ Introduction}

Computer aided object detection technology has a great contribution in automating effective medical diagnosis process. For example, the diagnostic imaging technology is considered as the most effective and handy way to examine the eye in a noninvasive way to the opthalmogists [1-2]. However, currently diabetes is the most rapidly spreading health dysfunction in the world [3-5]. In addition, related to the diabetes is diabetic retinopathy which is the most common effect of micro vascular changes in the retina. It is a major cause of blindness [3], [5-7]. Therefore, the ophthalmologists have adopted digital imaging system with automatic or semi automatic fundus image analysis algorithm for efficient screening [8]. But traditional 
digital RGB color images are limited in information for computerized analysis of retinal disorders. Apparently, due to this constraint multispectral imaging is getting more importance not only in color applications but also in other areas. Previous studies [4], [9-10] have comprehensively investigated the possibility, and effectiveness of photometric cues in retinal image analysis. Those investigations demonstrate that retinal spectral reflectance images provide more information to automatically detect the diseased lesions. The lesions are currently diagnosed by the ophthalmologists in terms of color and morphology. This study examined the strength of spectral imaging to analyze retinal images affected by diabetes. In this paper, we have proposed an effective approach to detect and classify abnormal lesions from spectral reflectance images of retina.

There exist some studies involving multispectral reflectance properties of ocular fundus [11-13]. But very few of them concentrate on detecting and classifying abnormal lesions on retinal surface. I.B. Styles et al. [12] found some cues to detect hemorrhages from retinal spectral reflectance images. However, our proposed method used retinal spectral image database called DIARETSPECDB1 [14] to analyze most of the diabetic retinopathies. In this study, we considered the same set of channels as features for all the retinopathies without the loss of generality. The addressed diabetic retinopathies are hemorrhages (Hem), hard exudates (HE), laser scars (LS), microaneurysms (MA) or red small dots (RSD), soft exudates (SE), intra retinal microvascular abnormalities (IRMA) and neovascularization (NV).

In general, the objects from spectral image can be detected or classified spectrally and/or spatially. Therefore, we considered a spectral image pixel vector as a random variable, and its spectrum as the probability distribution. In this way, stochastic measures could be applied to model the variability among the channels of two pixel vectors. Such similarity measures are spectral information measures (SIM), and spectral information divergence (SID), which are based on self-information theory and derived from the Kullback-Leibler information divergence [15]. They are very useful in hyperspectral image analysis to detect a particular target spectrum from a set of spectra. To do this, the relative spectral discriminatory probability (RSDBP) model is used. Adopting RSDPB, we developed the proposed method to use information encoded in the patterns of the reflectance. We also applied relative spectral discriminatory power (RSDPW) to evaluate the effectiveness of different spectral similarity measures to extract, and characterize the spectral properties of the training pixel vectors. Based on comparative analysis, we chose a suitable approach to find a representative of training class spectra. Using these reference spectra, we implemented several object detection methods used in hyperspectral imaging or multispectral magnetic resonance (MR) imaging. The methods are constrained energy minimization (CEM), orthogonal subspace projection (OSP) and Gaussian maximum likelihood (GML) [15].

Literatures relevant to retinal structures, RGB image analysis, retinal spectral imaging as well as several existing techniques used in multidimensional MR image analysis have been reviewed in section 2. Section 3 contains materials and proposed methods. Section 4 includes the experiments, results and comparisons. The conclusion has been made in section 5 , and section 6 contains direction for future work. 


\section{$2 \quad$ Literature Review}

Description of the complete structures of human eye and the retina, related to retinal diseases, is available in [4] and [16]. Retinal microvascular malfunctioning due to diabetes are discussed in [4], [6], and [17-18].

Tomi Kauppi [4] tried different possible ways to use RGB color information optimally to detect and classify diabetic retinopathy, and evaluated their performances. He separated the experiments by changing the color spaces, correcting illuminances and colors of the same set of retinal images, and also combined them. He concluded that photometric cues in RGB images are not good enough to detect and classify diabetic retinopathies in pixel levels. He recommended spectral retinal images for better performance.

P. Soliz et al. [10] studied the utility of spectral imaging of retinal ocular fundus to diagnose age related macular degeneration (ARMD). The authors mentioned about the possibility of developing noninvasive approach by using the light reflected from intraocular tissue to diagnose ARMD and diabetic retinopathy. However, it reported $54 \%$ classification accuracy by the weighted divergence based classification. Delori and Pflibsen [10-11] collected reflectance spectra from discrete points of retina to model the reflectance properties of anatomical tissue. The study concluded that the reflectance spectra were influenced by age, the transmission properties of the ocular media, discrete reflections in the stratified fundus layers, local melanin pigmentation, and the amount of blood in the choroid. Killbride and Keehnan [13] presented the spectral estimations to measure the spatial distribution of macular pigment in the retina using linear regression model.

I.B. Styles et al. [12] extensively described the technique to acquire multispectral retinal images, and quantitative analysis of them. They proposed a Monte Carlo model based method extracting the histological parameters to analyze the spectral reflectance images. The purpose of the research was to predict the retinopathy in earlier stages by analyzing the information collected from the model parameters. The parameters were corresponded to the retinal and choroidal haemoglobins, choroidal melanin, RPE melanin, and macular pigment.

Wang et al. [19] have proposed the method called Constrained Energy Minimization (CEM) for detection and classification of spectral target signatures in multispectral MR images. The method takes the advantage of spectral characteristics to detect the desired objects, and classify them. It has the ability to detect an object's spectra without having information of image background, and can be applied requiring only working knowledge of the desired object's signature spectra. It extracts the object of interest while minimizing the interfering effects induced from unknown sources including the image background. Lin et al. [20] have reported a great success to classify and segment three major tissues which are gray matter, white matter, and cerebral spinal fluid automatically from brain MR images extending CEM.

Harsanyi and Chang [21] developed Orthogonal Subspace Projection (OSP) based classifier to detect the spectral signature of an object from images. Each pixel vector, in this method, is projected onto a subspace which is orthogonal to the spectra of undesired objects. This operation may be considered as an optimal interference suppression process in the least squares sense. After minimizing the interfering i.e., undesired signatures, the residual is projected onto the signature of interest maximizing the 
signal-to-noise ratio. It results a single component image representing a classification map for the desired object. Wang et al. [22] have discussed different versions of OSP to analyze remotely sensed spectral images including MR images. It considers the MR image sequence as a multispectral image cube, and models each pixel vector as a linear mixture of tissue materials. The linear mixture model generates a fractional image for each object of interest considering a single pixel as a mixed pixel. Gaussian Maximum Likelihood (GML) estimations [15] are mainly based on a posteriori information obtained directly from image scene. This model is expressed in terms of a posteriori abundance estimation, and a posteriori noise estimation.

Basically the standard receiver operating characteristics (ROC) curve analysis tool [4], [23] is a frequently used method to evaluate the performance of two class classifiers. It is reliably used for diagnostic testing in medical community.

\section{$3 \quad$ Materials and Methods}

\subsection{Materials}

Collection of Spectral Images. Falt et al. [14] collected the set of spectral retinal images used in this project in collaboration of SIB labs in university of Eastern Finland and Kuopio University Hospital. The database called DIARETSPECDB1 is going to be publicly available with ground truths in future. The collected images were initially of 1024x1024 spatial resolution, and 31 spectral dimensions beginning from $400 \mathrm{~nm}$ to $700 \mathrm{~nm}$ with the interval $10 \mathrm{~nm}$. Fig. 1 shows some gray level images at different wavelengths and corresponding RGB image. One should notice Hem, RSD and LS visible in the image of abnormal eye at $550 \mathrm{~nm}$.
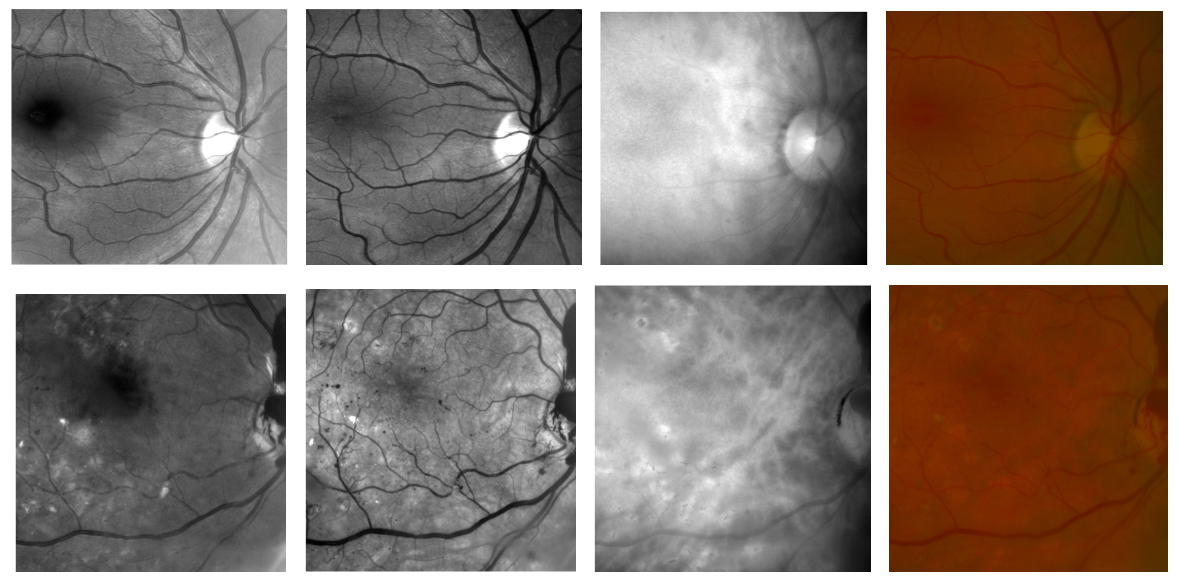

Fig. 1. Retinal spectral images (left to right): at $450 \mathrm{~nm}, 550 \mathrm{~nm}, 650 \mathrm{~nm}$, and RGB (upper row is of a normal eye, and the lower row is of an eye having retinopathy)

The used database contains in total 71 spectral images, among them 54 are from diabetic patients, and 17 are of non-diabetic control subjects. But there are varying number spectral channels in the images having at least last 25 channels [14]. 
In addition, we observed the flat responses of first six channels. Hence, we considered the last 21 channels in this study without the loss of generality. The captured retinal images contain diabetic retinopathies which are RSD, HE, SE, Hem, IRMA and NV.

Ground Truth. We collected the ground truths about diabetic retinopathies from the annotations made by three medical experts in department of ophthalmology, Kuopio University Hospital. We used the software toolbox IMGANNOTOOL [24] to label the annotations for the images. The author [4] has described the procedures to estimate or prepare such ground truths from multiple experts' annotations.

Training Data. The following pairs are the total number of images having a particular retinopathy and used training images: $\operatorname{Hem}(43,21), \operatorname{HE}(11,5), \operatorname{LS}(10,5)$, RSD $(42,21), \operatorname{SE}(3,2)$, IRMA $(2,1)$ and $\operatorname{NV}(3,2)$. That means we used around $50 \%$ of available images to collect training spectra for each retinopathy. From each training image, we collected about $50 \%$ of total spectra of a particular class. Also, to decorrelate from spatial dependencies, we collected the spectra randomly. So there were 7 training class for each of the 7 retinopathies with varying number of spectra in each class. Fig. 2 shows some spectra from each of the training class. Fig. 3 shows class representative spectra (mean and selected by RSDBP using SID similarity measure) needed for CEM, OSP and GML.
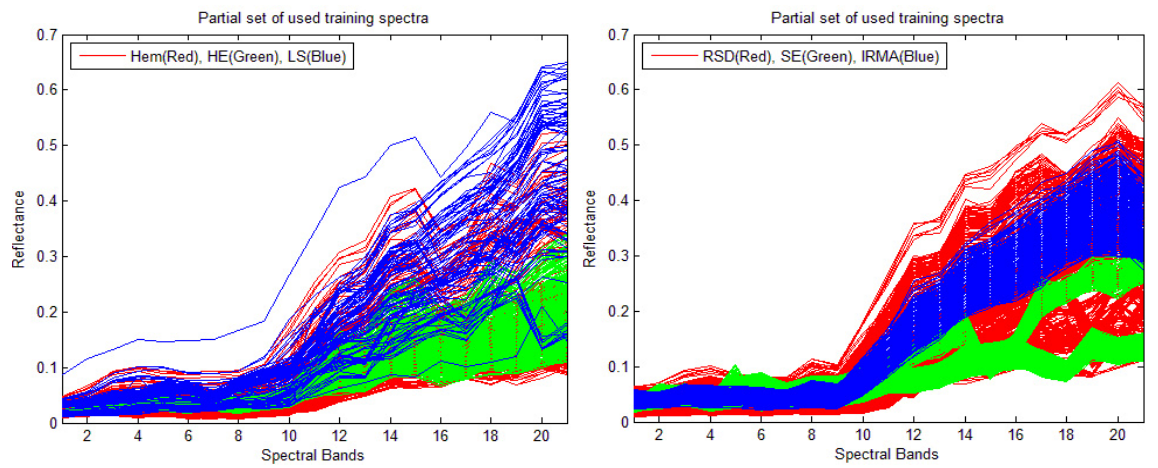

Fig. 2. (a b): Subset of training spectra for each retinopathy class: (a) Hem (red), HE (green), LS (blue), (b) RSD (red), SE (green), IRMA (blue)
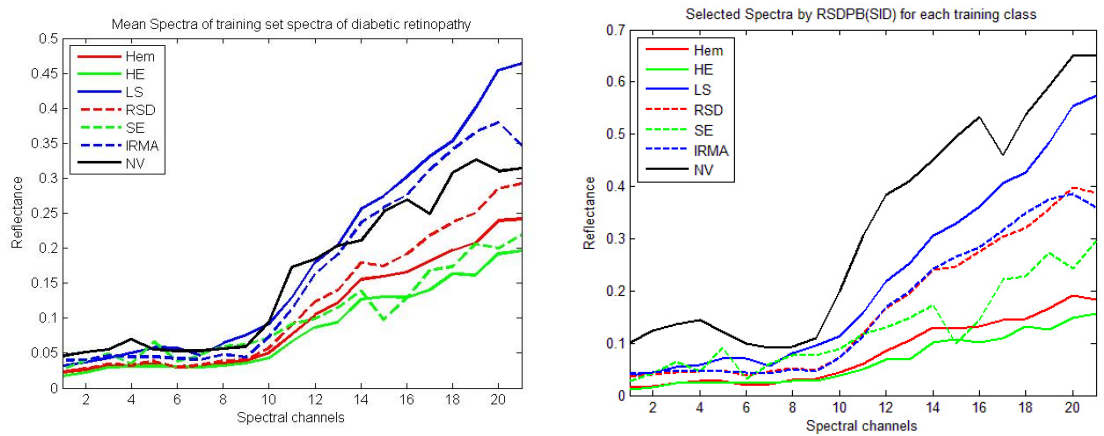

Fig. 3. (a b): (a) Mean spectra of training classes, (b) The most likelihood spectra chosen by RSDPB using SID 
Used Mathematical Models. We used SID for the first similarity measures between two spectral pixel vectors. This decision was based on experimental observations, and context of the spectral characteristics of the images. The assumption was that the sensitivity of spectral images could be better characterized by uncertainty. In this formulation, spectral band to band variability is considered as uncertainty resulting from randomness. Hence, SID is a stochastic modeling technique used to measure the spectral information [15]. It measures the discrepancy in probability distributions between two spectral vectors. In this technique, each pixel vector is treated as a random variable with the normalized spectra as the probability distribution. SID takes advantage of relative entropy which is also known as Kullback-Leibler information measure, directed divergence or cross entropy. It offers a new look of spectral similarity measures, and the detail mathematical formulation can be found in [15].

With the help of a similarity measure, the RSDPB model is used to recognize a target spectrum from a set of spectra. It measures the likelihood of a candidate spectrum to be the target. The mathematical formula for RSDPB calculates the discriminatory probabilities of all the spectra relative to a target spectrum to be identified. The candidate pixel vector can be accepted or rejected by applying a threshold on resulting probabilities. The mathematical description is also addressed in [15].

\subsection{Proposed Method}

Ideally, each type of object in the retina should be characterized by their reflectance properties with certain degree of variations. But in practice, we found large variations in spectra within a class, and very small or no variation with other class spectra (Fig. 2). We observed the class overlapping behavior of the spectra in both shape and magnitude. Beside, we found inaccurate or not effective to cover all the variations using one class representative spectrum (Fig. 3). These observations were confirmed by the analysis of the training spectra showing mixed pixel activity in the images. As a result, in most cases, very well established statistical classifiers failed to classify the training set [Table 1]. Moreover, large percentages of spectra were misclassified by the standard classifiers into other classes. This observation set the background to combine both stochastic and deterministic spectral similarity measures to develop the method. The approach is basically supervised, and so divided into training and testing module. In the training part, we collect the set of training spectra for each of 7 retinopathy classes. Then we calculate RSDPB using SID similarity measure considering class mean (Fig. 3a) as the reference spectrum. We extract another class parameter called normalization constant from RSDPB values and store all of them. The testing part follows the same calculations for each pixel using parameters of corresponding class. Finally, we define the class of the pixel as the class of training spectra with which the closest similarity is determined.

Pixel-wise Detection (two class classification). Basically, the proposed method works like a two class classifiers. The desired retinopathy is one class, and rests are in another class. The idea is to find the match of a test spectrum with any of the class to decide whether it is an abnormal pixel. This is done in two steps. In the first step, it 
finds an initial match evaluating the closeness of SID values between two pixel spectra. But SID produces one scalar value loosing information from band to band variation. So to make the decision more precise, we use a deterministic similarity measure (e.g. cross border distance (CBD)) to compare the geometric distance. However, the method needs as inputs the type of retinopathy to be detected, and threshold value. Then prediction or testing begins with this information as well as loaded training class parameters. The parameters come from the training phase which needs to be executed only once. The prediction module starts to apply the algorithm on each pixel of the input image. At the end of processing, it generates a binary mask containing $1 \mathrm{~s}$ in the pixels where it predicts the retinopathy.

Implementation. In the training phase, at first, we calculate the RSDPB values for each set of training class spectra, and store it. We use mean spectra as the reference spectra to calculate the SID values. However, the important point is that the same reference spectrum and normalized constant have to be used in testing phase. In the testing phase, for each pixel, the closeness of the RSDPB value with the values stored for training class is determined. We do it by interpolating RSDPB values of the corresponding training class. In this stage, the candidacy can be accepted or rejected. If accepted, then the output of this step is an index of a pixel spectrum in the training class. In this case, we know from which retinopathy class the index comes. In the second step, we calculate the geometric distance between the test, and the selected training pixel spectra. Spectral angle mapper (SAM) might be the most potential for testing the geometric similarity, but we applied simple CBD to avoid computational complexity. Finally, depending on the success of a threshold test, the pixel is defined as abnormal pixel, and assigned to the matched retinopathy class.

\section{Training Phase}

Input: Set of training class spectra for each diabetic retinopathy.

Output: Sorted arrays of relative spectral discriminatory probability (RSDPB) in a $2 D$ variable $\boldsymbol{P}$, and normalization constant in the array $\mathbf{N C}$ for each class.

1. for each of the retinopathy type $\mathbf{i}$ do

2. load corresponding training class spectra in $\mathbf{T}$

3. Calculate the mean spectra of $\mathbf{T}$ and assign to $\mathbf{M}$

4. for each spectra in the training class $\mathbf{T}$ do

5. Calculate spectral information divergence (SID) and store in TD

6. end for

7. Calculate the sum of array $\mathbf{T D}$ and assign in $\mathbf{N C}_{\mathbf{i}}$

8. for each element in the set TD do

9. Calculate RSDPB and store it in $\mathbf{P}_{\mathbf{i}}$

10. end for

11. Sort $\mathbf{P}_{\mathbf{i}}$ in ascending order retaining original indexes of the spectra

12. end for

13. Output $\mathbf{P}$ and $\mathbf{N C}$ 


\section{Testing Phase}

Input: $\boldsymbol{i}$ is the index of a spectral image in the database, $\boldsymbol{t}_{\mathbf{1}}, \boldsymbol{t}_{\mathbf{2}}$ are the threshold values, $\boldsymbol{r}$ is the index of chosen retinopathy type.

Output: img which is the RGB equivalent image of the input spectral image (with detected pixels in white color), and a $2 D$ binary mask containing 1's in detected locations (to calculate ROC parameters later).

1. Load TD, $\mathbf{P}$ and $\mathbf{N C}$ corresponding to the retinopathy type $\mathbf{r}$.

//Here, $\boldsymbol{T D}$ is the set of training class spectra, $\boldsymbol{P}$ and $\mathbf{N C}$ are the corresponding list of RSDPB and normalization constant for selected retinopathy type //

2. Load the spectral image $\mathbf{i}$ in $\mathbf{~ i m}$ and converted RGB image in img.

3. Create a same spatial sized binary image containing all zeros and call it mask.

4. Calculate the mean spectra of the training set $\mathbf{T D}$ and assign to $\mathbf{M}$.

5. for each pixel $\mathbf{p}$ in the image im do

6. Calculate SID using $\mathbf{M}$.

7. Calculate RSDPB from SID value and NC and assign to $\mathbf{p b}$.

8. Find the index ind of the closest spectra in the training set. Option-1: Interpolate the index in TD using the values pb and $\mathbf{P}$. Assign $\mathbf{- 1}$ to ind if it less than zero or greater than the maximum index in TD. Option-2: Subtract $\mathbf{p b}$ from $\mathbf{P}$, compare the lowest value in $\mathbf{P}$ with threshold $\mathbf{t}_{\mathbf{1}}$. If it passes the test assign to ind the index else assign -1 .

9. if ind is positive then do

10. load the spectra from TD in location ind and assign to tp

dist = geometric distance (e.g. CBD or ED) between the spectra $\mathbf{p}$ and $\mathbf{t p}$

11. if dist is less than $\mathbf{t}_{\mathbf{2}}$

12. Assign 255 to the pixel $\mathbf{p}$ in img and 1 to mask.

13. end if

14. end if

15. end for

16. Output img and mask.

\section{$4 \quad$ Experiments and Results}

\subsection{Experiments}

Training data classification. We applied different standard classifiers on the set of merged training spectra to have a look inside, and to check the applicability. Table-1 holds the result produced by LIBSVM [25] to classify the spectra. Note that the maximum accuracy achieved was less than 50\%. Because the linear kernel gave the better result, we applied linear discriminant analysis with more controls. The overall accuracy was $39.4 \%$ for parametric linear classifier, where a set of discriminant functions were used and the variables were statistically independent. Alternatively, the overall accuracy was $34.6 \%$, where the variables were assumed not statistically independent, and the covariance matrices were same for all. We did no additional scaling but normalized to make it zero-mean data. But all the tests produced very poor result. 
Table 1. Training data classification accuracy and MSE (\%) of SVM [25]

\begin{tabular}{|c|c|c|c|c|}
\hline & Linear kernel & Radial Basis Kernel & Sigmoid Kernel & Polynomial Kernel \\
\hline Accuracy & 42.03 & 40.3 & 35.19 & 31.6 \\
\hline MSE & 7.09 & 7.72 & 9.93 & 12.9 \\
\hline
\end{tabular}

Recall that there are four possible outcomes of a classifier with respect to a given instance. If both the instance and the output are positives or negatives, then it is counted as true positives (TP) or true negatives (TN) accordingly. If the instance is positive but the output is negative, then it is counted as false negatives (FN). If the instance is negative but the output is positive then it is counted as false positives (FP). Now true positive rate (tpr) or sensitivity $(\mathrm{SN})$ is: $(\mathrm{TP} /(\mathrm{TP}+\mathrm{FN}))$, specificity $(\mathrm{SP})$ is: $(\mathrm{TN} /(\mathrm{TN}+\mathrm{FP}))$, false positive rate (fpr) is: $(\mathrm{FP} /(\mathrm{FP}+\mathrm{TN}))$, accuracy is: $((\mathrm{TP}+\mathrm{TN}) /$ $(\mathrm{TP}+\mathrm{FP}+\mathrm{TN}+\mathrm{FN}))$, and $\mathrm{F}$-measure is: $(2 \mathrm{TP} /(2 \mathrm{TP}+\mathrm{FP}+\mathrm{FN}))$.

Test of existing multispectral object detection methods. We applied the methods CEM, OSP and GML on the whole database. As reference for each class, we used the spectra selected by RSDPB using SID values. We followed pixel wise classification strategy. Fig. 4 shows the ROC curves representing the detection performances. Each of the diagrams has 7 curves for 7 classes. Note that OSP and GML produced exactly same results for this dataset. So the comment for OSP also applies to GML.

Also recall that a realistic classifier has the ROC curve above the right diagonal line of the ROC space [4], [23]. If any classifier lies on the diagonal line which indicates that it classifies randomly with equal probability for positive and negative instances. If it lies below the line that means the classifier has not enough knowledge or incorrectly uses the information to classify the instances, and thus it fails. From the ROC curves in Fig. 4(a), it can be decided that CEM is not appropriate for this spectral dataset to use as a detector. Because for most of the diseased lesions, the ROC curves lay on or down of the right diagonal line. The method OSP (Fig.4 (b)) is though not sufficient but better than CEM. Because some parts of the OSP produced ROC curves are below, and other parts are above the right diagonal line.
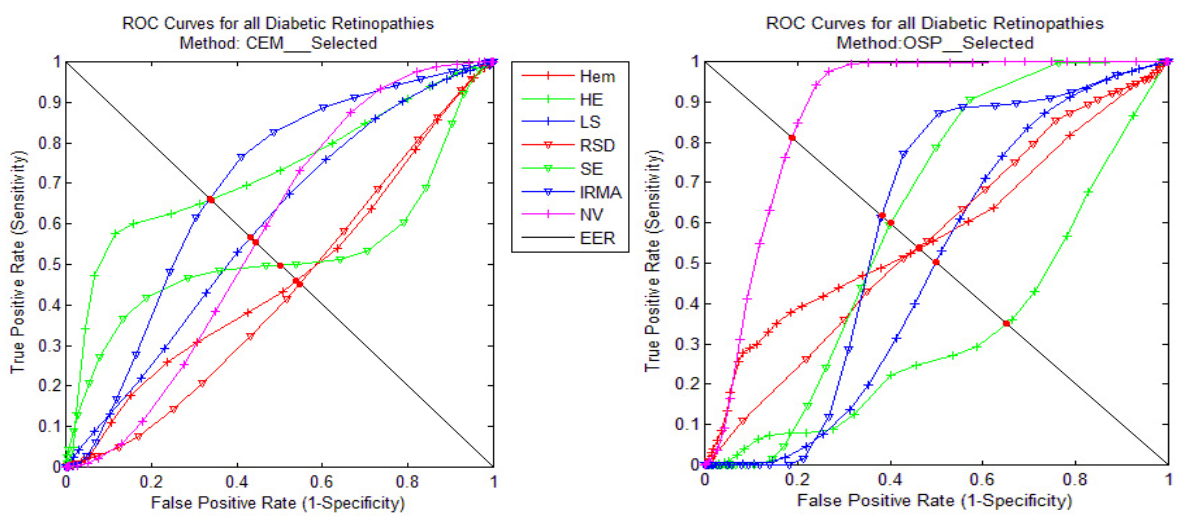

Fig. 4. (a, b): ROC curves: (a) CEM (b) OSP/GML 


\subsection{Results of the Proposed Method}

According to the comments made, in just previous subsection, the proposed method is successful satisfying all pragmatic issues, because the ROC curves (Fig. 5) for all the retinopathies rest above the right diagonal line. In fact, all the ROC curves comfortably lay above the line, except for hemorrhages, due to its position just over the diagonal line. It means the classifier has not enough information to classify all the pixels in hemorrhages lesions.

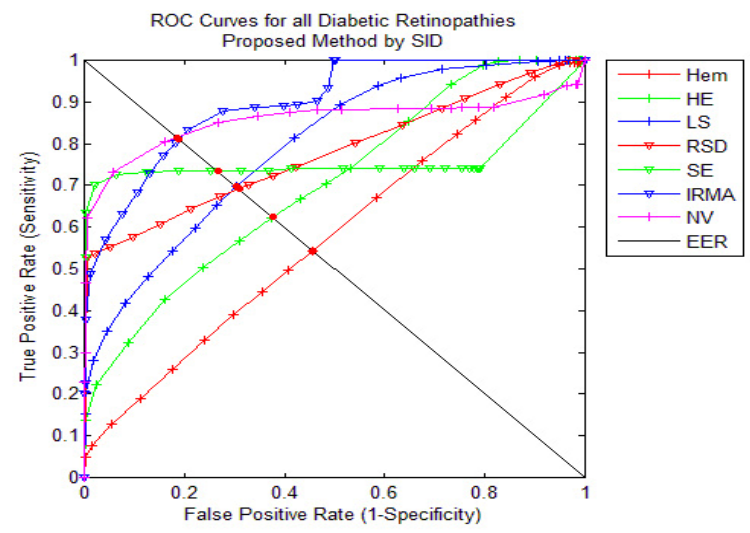

Fig. 5. ROC performance curves of the proposed method

We estimated single pair of sensitivity and corresponding specificity within 95\% confidence interval to make it reasonable. The estimation was performed according to Bayes theorem [26]. Table 2 contains all these quality measures for the proposed method.

Table 2. ROC curve data analysis of the proposed method

\begin{tabular}{|c|c|c|c|c|c|}
\hline \multirow{2}{*}{ Retinopathy } & \multirow{2}{*}{$\begin{array}{c}\text { Equal } \\
\text { Error Rate } \\
\text { (EER) }\end{array}$} & \multirow{2}{*}{$\begin{array}{c}\text { Area Under } \\
\text { the Curve } \\
\text { (AUC) }\end{array}$} & \multicolumn{2}{|c|}{$\begin{array}{c}\text { Performance } \\
\text { (95\% Confidence Interval) }\end{array}$} & \multirow[b]{2}{*}{ p-value } \\
\hline & & & $\begin{array}{l}\text { Sensitivity } \\
\text { (SN) }\end{array}$ & $\begin{array}{l}\text { Specificity } \\
\text { (SP) }\end{array}$ & \\
\hline Hem & 0.457 & 0.577 & 0.577 & 0.577 & 0.000 \\
\hline $\mathrm{HE}$ & 0.376 & 0.703 & 0.703 & 0.703 & 0.000 \\
\hline $\mathrm{LS}$ & 0.303 & 0.793 & 0.792 & 0.793 & 0.000 \\
\hline RSD & 0.308 & 0.778 & 0.778 & 0.779 & 0.000 \\
\hline SE & 0.266 & 0.763 & 0.761 & 0.764 & 0.000 \\
\hline IRMA & 0.188 & 0.898 & 0.898 & 0.899 & 0.000 \\
\hline $\mathrm{NV}$ & 0.185 & 0.856 & 0.856 & 0.857 & 0.000 \\
\hline Average & 0.298 & 0.767 & 0.766 & 0.767 & N/A \\
\hline
\end{tabular}


The average EER is 0.298 which is around $30 \%$. It reflects that $30 \%$ pixels have equal probability to be classified as either normal or abnormal, irrespective of their original class. So for those $30 \%$ pixels, the classifier did not have sufficient information to correctly classify them. Though estimation technique is acceptable enough but exact values of these parameters (Table 3) should give more confidence to the readers. For this purpose, we chose the best cut-off point on a ROC curve. It is the point closest to the left and upper extreme point of the ROC space [23].

Table 3. Performances at the best cut-off point of the proposed method

\begin{tabular}{|c|c|c|c|c|c|c|c|}
\hline Retinopathy & $\begin{array}{c}\text { Cut- } \\
\text { Off } \\
\text { Value }\end{array}$ & $\begin{array}{c}\text { Pixel-wise } \\
\text { Prevalence } \\
(\%)\end{array}$ & $\begin{array}{c}\text { Sensitivity } \\
(\text { SN \%) }\end{array}$ & $\begin{array}{c}\text { Specificity } \\
(\text { SP \%) }\end{array}$ & $\begin{array}{c}\text { F- } \\
\text { Measure } \\
(\%)\end{array}$ & $\begin{array}{c}\text { Accuracy } \\
(\%)\end{array}$ & $\begin{array}{c}\text { Miss } \\
\text { classification } \\
\text { Rate (\%) }\end{array}$ \\
\hline Hem & 2.10 & 1.72 & 54.23 & 54.37 & 3.92 & 54.36 & 45.64 \\
\hline HE & 4.00 & 0.43 & 62.12 & 62.77 & 1.40 & 62.77 & 37.23 \\
\hline L S & 2.10 & 0.11 & 69.90 & 69.53 & 0.51 & 69.53 & 30.47 \\
\hline RSD & 5.00 & 0.07 & 64.37 & 78.84 & 0.40 & 78.83 & 21.17 \\
\hline SE & 7.00 & 0.02 & 72.42 & 93.79 & 0.40 & 93.79 & 6.21 \\
\hline IRMA & 1.90 & 0.06 & 83.11 & 79.49 & 0.48 & 79.49 & 20.51 \\
\hline NV & 5.00 & 0.07 & 80.45 & 83.97 & 0.70 & 83.97 & 16.03 \\
\hline Average & N/A & $\mathbf{0 . 3 5}$ & $\mathbf{6 9 . 5 1}$ & $\mathbf{7 4 . 6 8}$ & $\mathbf{1 . 1 2}$ & $\mathbf{7 4 . 6 8}$ & $\mathbf{2 5 . 3 2}$ \\
\hline
\end{tabular}

The average occurrence of abnormal pixels is just $0.35 \%$ (Table 3 ) for the used database. The specificity value for red small dots (RSD) indicates that the proposed method can differentiate $78.84 \%$ of normal pixels as normal. It is important because RSDs are the smallest and most frequently occurring retinopathy. The proposed algorithm performed poor in case of recognizing pixels from hemorrhages regions. One reason was that some ground truths regarding this disease had incorrect information. They affected both training and evaluation phases. The natural large variation in spectra due to color and density might be another cause of poor accuracy. Next to hemorrhages, the low performance was for hard exudates (around 63\%). The reason might be unusual variations in spectral shapes. The accuracy (almost 79\%) of RSDs detection is good.

\subsection{Comparisons}

Multidimensional object detection methods. Table 4 contains EER estimated from the ROC curves for all the discussed multispectral methods. The EER should be less than 0.5 for a classifier to be standard [23], [4]. So the EER (shaded cells) above 0.5 demonstrates the corresponding method should not be considered as classifiers for that retinopathy. Bolded values indicate the best performances i.e. the smallest EER. Note OSP and GML produced exactly the same performances. 
Table 4. Equal Error Rate (EER) of all methods

\begin{tabular}{|c|c|c|c|c|}
\hline Retinopathy & Proposed method & CEM & OSP & GML \\
\hline Hem & $\mathbf{0 . 4 5 7}$ & 0.540 & 0.463 & 0.463 \\
\hline HE & 0.376 & $\mathbf{0 . 3 4 0}$ & 0.651 & 0.651 \\
\hline LS & $\mathbf{0 . 3 0 3}$ & 0.433 & 0.498 & 0.498 \\
\hline RSD & $\mathbf{0 . 3 0 8}$ & 0.547 & 0.461 & 0.461 \\
\hline SE & $\mathbf{0 . 2 6 6}$ & 0.502 & 0.400 & 0.400 \\
\hline IRMA & $\mathbf{0 . 1 8 8}$ & 0.337 & 0.382 & 0.382 \\
\hline NV & $\mathbf{0 . 1 8 5}$ & 0.445 & 0.188 & 0.188 \\
\hline Average & $\mathbf{0 . 2 9 8}$ & 0.449 & 0.435 & 0.435 \\
\hline
\end{tabular}

Table 5 contains another performance measures called area under the curve (AUC) produced by the ROC curves. If a classifier guesses randomly then its AUC will be equal or less than 0.5. So the AUC should be no less than 0.5, if a classifier is to be successful [23]. In Table 5, the values are shaded which have AUC less than 0.5. The values are bolded which represent the best performances (highest AUC).

Table 5. Area under the curve (AUC) of all methods

\begin{tabular}{|c|c|c|c|c|}
\hline Retinopathy & $\begin{array}{c}\text { Proposed } \\
\text { method }\end{array}$ & CEM & OSP & GML \\
\hline Hem & 0.577 & 0.419 & $\mathbf{0 . 5 7 9}$ & $\mathbf{0 . 5 7 9}$ \\
\hline HE & $\mathbf{0 . 7 0 3}$ & 0.573 & 0.335 & 0.335 \\
\hline LS & $\mathbf{0 . 7 9 3}$ & 0.519 & 0.488 & 0.488 \\
\hline RSD & $\mathbf{0 . 7 7 8}$ & 0.498 & 0.557 & 0.557 \\
\hline SE & $\mathbf{0 . 7 6 3}$ & 0.437 & 0.621 & 0.621 \\
\hline IRMA & 0.898 & $\mathbf{0 . 9 0 0}$ & 0.599 & 0.599 \\
\hline NV & 0.856 & 0.492 & $\mathbf{0 . 8 7 9}$ & $\mathbf{0 . 8 7 9}$ \\
\hline Average & $\mathbf{0 . 7 6 7}$ & 0.548 & 0.580 & 0.580 \\
\hline
\end{tabular}

From the comparisons, it can be concluded that CEM, OSP and GML fail as classifiers for some of the retinopathies. But the proposed method is successful for all the retinopathies.

$R G B$ image analysis methods. To avoid unambiguity, it should be reminded that the following is a coarse comparison. Because to compare two methods, it is typical to apply them on the same dataset, but in this case, the datasets were different. Still we compared to have an approximate idea of the performances of spectral approach and RGB image based approach. However, we considered the results from Ref. 4 which aimed to find the optimum performances of RGB photometric cues in analyzing diabetic retinopathy. The research [4] addressed four types of retinopathies (Table 6). We derived the average EER from the tabulated values mentioned in [4]. Bolded values show the better performances. 
Table 6. EER of RGB image analysis method [4] and proposed method

\begin{tabular}{|c|c|c|c|c|c|}
\hline & Hem & RSD & HE & SE & Avg. \\
\hline $\begin{array}{c}\text { Traditional RGB method } \\
\text { (Average of Test \& Training data) }\end{array}$ & $\mathbf{0 . 3 7 5}$ & 0.34 & $\mathbf{0 . 3 6}$ & 0.34 & 0.35 \\
\hline $\begin{array}{c}\text { Proposed spectral method } \\
\text { (Test + Training data) }\end{array}$ & 0.46 & $\mathbf{0 . 3 1}$ & 0.38 & $\mathbf{0 . 2 7}$ & 0.35 \\
\hline
\end{tabular}

The author [4] also reported the performances in terms of symmetry points where sensitivity is equal to specificity on ROC curve. Table 7 shows the average performances of different experiments done in the study [4]. As the units are now symmetry points, so the larger value indicates the better performance (bolded values).

Table 7. Performances in terms of symmetry points

\begin{tabular}{|l|c|c|c|c|c|}
\hline & Hem & RSD & HE & SE & Avg. \\
\hline Traditional RGB method (Average) & $\mathbf{0 . 6 4}$ & 0.65 & $\mathbf{0 . 6 8}$ & 0.62 & 0.65 \\
\hline Proposed spectral method & 0.54 & $\mathbf{0 . 6 9}$ & 0.62 & $\mathbf{0 . 7 3}$ & 0.65 \\
\hline
\end{tabular}

\section{Conclusions}

Spectral imaging and reproduction can explore detailed information of object surface than traditional 3-channel (e.g. RGB, CMY) imaging. Also, following spectral approach, the influence of spectral characteristics of imaging systems, illuminations, viewing environments etc. can be significantly reduced. Hence, applications of spectral imaging are growing in many areas. One particular area is medical image analysis. However, the main purpose of this study was to develop method to detect and classify diabetic retinopathies from spectral images, and thus find the prospect of spectral imaging. In this paper, we have proposed a method which is basically a spectral reflectance pattern matching technique towards this objective. The proposed method is simultaneously a detector and classifier. It works as a two class classifiers using both stochastic and deterministic spectral similarity measures. In the training phase, the method collects necessary information to characterize a class of spectra, and in testing phase, this information is used to define the class of a pixel vector. Experimental results show some success in analyzing various diabetic retinopathies. In addition, we evaluated existing multidimensional object detection techniques used both in remotely sensed and medical image analysis. Though, in general, they provided worst results, but OSP or GML could be studied further. In addition, we roughly compared the results with traditional but optimized RGB image analysis methods. But we found little difference of their overall performances. The performance of the proposed method should be improved by applying advanced feature extraction and selection procedures. The result in this study, thus, investigates the basic potency of spectral imaging. Besides, the images were not full free from spectral errors. Hence, initial emphasis has to be given in collecting retinal spectral images to circumvent all possible errors, especially, due to involuntary eye movements, and thus in image registrations. 


\section{$6 \quad$ Future Work}

To define the class accurately, the test pixel vector can be compared with all the training classes, instead of, comparing only with the selected retinopathy. Another idea to improve the accuracy is to discard the pixels contained in the normal components like optic disc, macula and vessels, and then apply the detection methods. Other direction is to use Hidden Markov Model-Based Information Divergence (HMMID) to extract the probabilistic divergence between two vectors. Alternative to supervised approach could be unsupervised vector quantization (UVQ) or unsupervised target generation process (UTGP) [15]. Moreover, the RX algorithm developed by Reed and Yu, and the low probability detection (LPD) model [15] could be interesting to apply and see the performances.

Acknowledgements. The authors thank the Finnish Funding Agency for Technology and Innovation (TEKES Project 40039/07), and the partners of the ImageRet project (http://www.it.lut.fi/project/imageret/) for their support. Medical experts and other researchers were involved from Kuopio University Hospital and Lappenranta Technical University to collect images and provide medical information. The authors also thank Lasse Lensu, Pauli fält,Tomi Kauppi, Joni-Kristian Kamarainen, Heikki Kälviäinen and Hannu Uusitalo who contributed in collecting the original data and expert annotations. This study was a thesis of EU Erasmus Mundus Joint Master Degree program called CIMET (Color in Informatics and Media Technology).

\section{References}

1. Kuivalainen, M.: Retinal Image Analysis using Machine Vision, Masters Thesis. Lappeenranta University of Technology (June 6, 2005)

2. von Wendt, G.: Screening for diabetic retinopathy: Aspects of photographic methods. PhD thesis, Karolinska Institutet (2005)

3. World Health Organization, Diabetes action now: An initiative of the world health organization and the international diabetes federation, nr ISBN 924159151 (2004)

4. Kauppi, T.: Eye Fundus Image Analysis for Automatic Detection of Diabetic Retinopathy. PhD Thesis, Lappeenranta Technical University (2010)

5. Carinci, F., Stracci, F., Storms, F.: Diabetes. In: Major and Chronic Diseases, Report 2007 (2007)

6. Gardner, T.W., Antonetti, D.A., Barber, A.J., LaNoue, K.F., Levison, S.W.: Diabetic Retinopathy: More Than Meets the Eye. Survey of Opthalmology 47(2), 253-262 (2002)

7. Diabetes mellitus, http://en.wikipedia.org/wiki/Diabetes (accessed May 7, 2011)

8. Abramoff, M.D., Niemeijer, M., Suttorp-Schulten, M.S., Viergever, M.A.: Evaluation of a system for automatic detection of diabetic retinopathy from color fundus photographs in a large population of patients with diabetes. Diabetes Care 31(2), 193-197 (2008)

9. Rahaman, G.M.A., Ahsan Rajon, A.H.M., Rahman, A.: Effective Approach for Automatic Detection of Vessels, Optic Disk and Macula: Retinal Spectral Image Analysis Perspective. International Journal of Applied Research in Computer Science and Information Technology (IJAR-CSIT), 25-37 (2012) 
10. Soliz, P., Truitt, P., Nemeth, S.: Spectrally-Based Fundus Imaging: Implications for Image Enhancement And Diagnosis of Retinal Diseases. In: Thirty-Fifth Asilomar Conference on Signals, Systems and Computers, Novemer 4-7, vol. 2, pp. 1268-1272. IEEE Explore (2001)

11. Delori, F., Pflibsen, K.: Spectral reflectance of the human ocular fundus. Applied Optics 28(6), 1061-1077 (1989)

12. Styles, I., Calcagni, A., Claridge, E., Orihuela-Espina, F., Gibson, J.: Quantitative analysis of multi-spectral fundus images. Medical Image Analysis 10(4), 578-597 (2006)

13. Kilbride, P.E., Keehan, K.M.: Visual pigments in the human macula assessed by imaging fundus reflectometry. Applied Optics 29(10), 1427-1435 (1990)

14. Falt, P., Hiltunen, J., Hauta-Kasari, M., Sorri, I., Kalesnykiene, V., Uusitalo, H.: Extending Diabetic Retinopathy Imaging from Color to Spectra. In: Proceedings of the Scandinavian Conference on Image Analysis (SCIA 2009), Oslo, Norway (2009)

15. Chang, C.-I.: Hyperspectral Imaging: Techniques for Spectral Detection and Classification, 1st edn. Springer (2003)

16. Eye Anatomy and Function, http://faculty.washington.edu/chudler/ eyetr.html (accessed May 11, 2011)

17. Diabetic Eye Disease, http: / / www. emedicinehealth.com/ diabetic_eye_disease/article_em.htm\#DiabeticEyeDiseaseOverview (accessed May 12, 2011)

18. Diabetic Retinopathy http://en.wikipedia.org/wiki/ Diabetic_retinopathy (accessed May 7, 2011)

19. Wang, C.-M., Chen, C.-C., Chung, N., Yang, C., Chung, P.-C., Yang, C.-W., Chang, C.-I.: Detection of Spectral Signatures in Multispectral MR Images for Classification. IEEE Transactions on Medical Imaging 22(1), 50-61 (2003)

20. Lin, G.-C., Wang, W.-J., Wang, C.-M.: Feature Selection Algorithm for Classification of Multispectral MR Images Using Constrained Energy Minimization. In: 10th International Conference on Hybrid Intelligent Systems, Atlanta, GA, August 23-25, pp. 43-46. IEEE Xplore (2010)

21. Harsanyi, J., Chang, C.-I.: Hyperspectral image classification and dimensionality reduction: an orthogonal subspace projection approach. IEEE Transactions on Geoscience and Remote Sensing 32(4), 779-785 (1994)

22. Wang, C., Yang, S., Chung, P., Chang, C., Lo, C., Chen, C., Yang, C., Wen, C.: Orthogonal Subspace Projection-based Approaches to Classification of MR image Sequence. Computerized Medical Imaging and Graphics 25(6), 465-476 (2001)

23. Fawcett, T.: An introduction to ROC analysis. Pattern Recognition Letters 27, 861-874 (2009)

24. IMAGERET, http://www2.it.lut.fi/project/imageret/ (accessed June 08, 2011)

25. Chang, C.-C., Lin, C.-J.: LIBSVM : a library for support vector machines (2001), http://www.csie.ntu.edu.tw/ cjlin/libsvm/

26. Cardillo, G.: Clinical test performance: the performance of a clinical test based on the Bayes theorem, http: / /www. mathworks. com/matlabcentral/fileexchange/ 2705-clinical-test-performance (accessed May 11, 2011) 